\title{
Prognostic performance of different lymph node staging systems after curative resection for Siewert type II adenocarcinoma of the esophagogastric junction
}

\author{
Weilin Zhang ( $035847428 @ q q . c o m$ ) \\ Hunan Provincial People's Hospital https://orcid.org/0000-0001-6482-9895 \\ Yong Li \\ Southern Medical University Nanfang Hospital
}

\section{Research}

Keywords: Adenocarcinoma of the esophagogastric junction, Log odds of positive lymph nodes, Lymph node ratio, Prognosis

Posted Date: August 12th, 2020

DOI: https://doi.org/10.21203/rs.3.rs-56058/v1

License: () (1) This work is licensed under a Creative Commons Attribution 4.0 International License. Read Full License 


\section{Abstract}

Background: The tumor-node-metastasis (TNM) pN stage, which is based on the number of positive lymph nodes (LNs), is an important prognostic factor for patients with adenocarcinoma of the esophagogastric junction (AEG). The lymph node ratio (LNR) and log odds of metastatic lymph nodes (LODDS) staging systems are new effective indicators of prognosis. We aimed to evaluate their prognostic value in Siewert type II AEG.

Methods: Patients diagnosed with Siewert type II AEG who underwent curative resection between 2004 and 2014 at Guangdong General Hospital were recruited. A Cox regression model was constructed, and prognostic performance was measured using Harrell's concordance index (C-index) and the Akaike information criterion (AIC).

Results: When LN status was modeled as a continuous variable, the LODDS system (C-index: 0.729; AIC: 940.483) outperformed the other staging systems, including the number of positive LNs (LNP) (C-index: 0.721; AIC: 946.935) and LNR (C-index: 0.725; AIC: 938.918).However,when assessed as categorical variables, the LNR staging system had a better prognostic performance (C-index: 0.752; AIC: 926.350) than the American Joint Committee on Cancer (AJCC) 8th edition TNM pN (C-index: 0.740; AIC: 934.349) and LODDS (C-index: 0.737; AIC: 939.087) staging systems. Each LNR stage is more evenly distributed than the other two staging systems. Moreover, the LODDS stage is more dependent on the TLNE.

Conclusion: The LNR represented the best prognostic factor when assessed as a categorical variable and may serve as an alternative nodal staging system for AEG.

\section{Introduction}

There has been an increasing incidence of adenocarcinoma of the esophagogastric junction (AEG) worldwide over the past several decades[1-3]. AEGs are defined as tumors that have centers located within $5 \mathrm{~cm}$ proximal and distal to the anatomical cardia and are divided into three distinct tumor entities (types IIII)[4]. As with other high-risk gastrointestinal cancers, the number of lymph node (LN) metastases is an important prognostic indicator for AEG[5]. In 2016, the American Joint Committee on Cancer (AJCC) released the 8th edition of the tumor-node-metastasis (TNM) classification, in which Siewert type II AEG was staged as esophageal carcinoma[6]. However, it has been reported that the current TNM pN staging system can be influenced by the total number of LNs examined (TNLE) and might cause stage migration[7, 8]. To provide a more accurate LN staging system, other indicators have been proposed.

The lymph node ratio (LNR) is the ratio of positive LNs to TNLE. It represents the "probability" of LN metastasis. Two positive LNs over four examined is equal to $4 / 8,5 / 10$, etc.[9] and has the ability to avoid the stage migration phenomenon[10]. Several authors have proposed that the LNR system is an independent prognostic factor and has better prognostic value than the TNM pN stage in patients with resected gastric cancer[11, 12]. The log odds of metastatic lymph nodes (LODDS) is the natural logarithm derived from the ratio of positive LNs + 0.5/negative LNs + 0.5[13]. Compared with the LNR systems in pNO disease, the LODDS score is more sensitive to TNLE. The superiority of the LODDS score as a prognostic predictor has 
been validated in patients with various malignancies, including ggastric cancer[11], esophageal cancer[14], breast cancer[15], colorectal cancer[16] and others. However, some studies did not note any superiority of the LODDS score over the TNM pN stage or LNR[17, 18].

Until now, data on the role of the LNR and LODDS score in the prediction of overall survival (OS) for AEG have been limited. In the present study, we aimed to compare the prognostic performance of the three LN staging systems in Siewert II AEG patients undergoing curative resection.

\section{Patients And Methods}

We identified patients undergoing curative resection for Siewert II AEG from 2004 to 2014 at Guangdong General Hospital. Patients who underwent palliative surgery, with stage IV disease according to the AJCC/UICC classification[19], or who underwent neoadjuvant therapies were excluded. The lymphadenectomy strategy was determined using the recommendations of the Japanese Research Society for Gastric Carcinoma[20]. The final dataset included 157 patients. Patients were classified with the three LN staging systems (TNM pN, LNR and LODDS) based on pathological results. The TNM pN stage was defined according to the 8th edition of the UICC/AJCC TNM staging system as follows: N0, negative; N1, 1-2 positive LNs; N2, 3- 6 positive LNs; and N3, > 6 positive LNs. The LNR was defined as the ratio between the number of metastatic lymph nodes and the TNLE. After reviewing the literature on AEG, we chose the LNR classification system with interval values of $0,0-0.3,0.3-0.6$, and $0.6-1$ [21]. The LODDS score was classified according to the method described by Chen et al.[22] as follows: LODDS1 (-2.11 $\leq$ LODDS1 $\leq-1)$, LODDS2 $(-1<$ LODDS2 $\leq-0.5)$, LODDS3 $(-0.5<$ LODDS3 $\leq 0)$ and LODDS4 $(0<$ LODDS4 $\leq 1.61)$.

OS was calculated on the basis of the Kaplan-Meier method and compared by the log-rank test. Spearman's correlation analysis was performed to assess the correlation between the TNLE, number of positive LNs (LNP), LNR and LODDS score. All significant variables in the univariate analysis were included in the multivariate analysis. We performed Cox multivariate regression analyses to test whether each of the LN staging systems was an independent prognostic factor for survival. Harrell's C-index statistic (C-index) and the Akaike information criterion (AIC) were used to evaluate the discriminatory ability of the models of the three LN staging systems. The C-index ranged from 0.5 (no discrimination) to 1 (perfect discrimination), and the highest value was chosen as the best prognostic prediction model[23]. For the AIC, the lower the value was, the more accurate the model[24]. Based on the UICC/AJCC TNM staging system, we chose 16 as the cutoff value and compared the performance of the three LN staging systems under different LN dissection levels. The accepted level of statistical significance was defined as $P<0.05$. The statistical analysis was performed using the SPSS 23.0 statistical package (SPSS, Inc., Chicago, IL) and the R programming language (version 3.2.2 for Windows).

\section{Results}

\section{Patient characteristics}


The baseline characteristics of the 157 Siewert type II AEG patients are shown in Table 1. There were 111 (70.7\%) males and 46 (29.3\%) females. The median age was 62 years, ranging from $33 \sim 83$ years. The median tumor size was $4.5 \mathrm{~cm}$ (range, $1.3 \sim 12.0 \mathrm{~cm}$ ). With respect to surgery, 98 patients underwent curative proximal gastrectomy, and 59 patients underwent total gastrectomy. According to histopathological grading, well and moderately differentiated tumors were observed in 94 (59.9\%) patients, and poorly differentiated or undifferentiated tumors were observed in the remaining $63(40.1 \%)$ patients. The mean and median total number of lymph nodes examined (TLNE) were 18.6 and 17.0, respectively, with a range of 164 and a standard deviation (SD) of 10.7. There were 115 (73.2\%) patients with LN metastasis, and 42 (26.8\%) were free of nodal disease. The median LNR of the entire population was 0.16 . The LODDS values ranged from -2.11 to 1.61 , with a median value of -0.56 . In the Kaplan-Meier analysis, age, histological grade, TNM pT stage, tumor size, lymphovascular invasion, TNLE and each of the LN staging systems were significantly correlated with OS.

\section{Survival}

The median follow-up time for all patients was 42 months. The 5-year OS rate for the whole group of patients was $39.5 \%$, and the median OS time was 42 months. The Kaplan-Meier curves of the OS of patients according to the TNM pN stage, LNR, and LODDS score are shown in Fig. 1. For the TNM pN staging system, the 5 -year OS rates were $69.0 \%, 32.6 \%, 41.2 \%$, and $11.4 \%$, respectively $(P<0.001)$. For the LNR staging system, the 5 -year OS rates were $69.0 \%, 44.4 \%, 46.5 \%$, and $9.3 \%$, respectively $(P<0.001)$. For the LODDS staging system, the 5 -year OS rates were $70.8 \%, 56.0 \%, 35.8 \%$, and $7.4 \%$, respectively $(P<0.001)($ Table 1$)$. However, the survival curves of each LN staging system overlapped. Patients in pN1 stage had higher survival rates than patients in pN2 stage. One possible explanation for this finding is that some pN2 patients were incorrectly staged as pN1 due to an insufficient TLNE, which is defined as stage migration. All of the variables that were statistically significant in the univariate survival analysis were entered into the multivariate survival analysis.

\section{Performance of the LN classification systems}

Through Cox regression modeling, the LN staging system with the best prognostic discriminatory ability was assessed through iterative statistical models and through the comparison of C-index and AIC values. The TNM pN stage, LNR and LODDS systems all showed significant prognostic value in the multivariate Cox regression analyses. When assessed as categorical variables, the LNR staging system had a better prognostic performance (C-index: 0.752; AIC: 926.350) than the AJCC 8th edition (C-index: 0.740; AIC: 934.349) and LODDS (C-index: 0.737; AIC: 939.087) staging systems. When stratified by the TLNE, the LNR remained the best performing model among patients with less than 16 retrieved LNs (C-index: 0.810; AIC: 352.293). When LN status was modeled as a continuous variable, the LODDS scoring system (C-index: 0.729; AIC: 940.483) outperformed other scoring systems, including the LNP (C-index: 0.721; AIC: 946.935) and LNR (C-index: 0.725; AIC: 938.918) (Table 3). However, among patients with less than 16 LNs examined, the LNP system performed the best (C-index: 0.809; AIC: 350.265). 
The results of Spearman's correlation analyses are shown in Table 3. We found that both the LNP $(r=0.198$ $P=0.013)$ and LODDS $(r=-0.234 P=0.003)$ values correlated with the TLNE. However, no significant correlations between the LNR and TLNE were observed. There were significant and positive correlations between the LNR and LNP $(r=0.927 P=0.000)$, between the LODDS score and LNP $(r=0.887 P=0.000)$, and between the LODDS score and LNR v $(r=0.965 \mathrm{P}=0.000)$.

We created scatter plots to explain the relationship between the LNR and LODDS score. As shown in Fig. 2, the LODDS value increased with the LNR, indicating a close correlation. However, when the LNR was close to 0 or 1 , the LODDS system was able to efficiently distinguish heterogeneity. The relation of the TNM pN stage, LNR and LODDS staging systems differs according to the TLNE (Fig. 3). The LNR staging system includes stages 1-4 for 6 LNs examined, and there is no stage 1 in the LODDS system for less than 16 LNs examined. The LODDS classification in stage 3 has 24 LNs examined 3-13 metastatic LNs examined and 3-9 metastatic LNs by 16 LNs examined. Therefore, the LODDS system does not perform well with a relatively large TLNE.

\section{Discussion}

The extent of gastrectomy and lymphadenectomy for Siewert II AEG remains controversial[25-27]. To some extent, it has caused a difference in the distribution of the number of retrieved LNs at different institutions. Hasegawa et al. reviewed patients who had AEG types II/III and underwent D1 or more radical lymphadenectomy and found that the total number of LNs was > 15 in 153 patients but < 14 in 10 patients. Wu et al.[28] studied 1302 Siewert type II AEG patients through the Surveillance, Epidemiology, and End Results Program (SEER) database, of which 423 (32.49\%) patients had less than 10 TLNE. Our data showed that $45.2 \%$ of patients had less than 16 LNs examined, and the mean TLNE was $18.6 \pm 10.7$. This result is consistent with the TLNE described by Liu et al[29] (16.6 \pm 9.1$)$, who evaluated changes in AEG among surgical patients from 1988 to 2012 in a high-volume Chinese hospital. These data indicate that inadequate LN dissection is common in AEG, and stage migration may often be overlooked using the current TNM pN staging system. Thus, it is necessary to explore an LN staging system with more prognostic value.

Our study demonstrated that both the LNR and LODDS staging systems were superior to the traditional lymph node staging system. When assessed as categorical variables, the LNR outperformed the LODDS and TNM pN staging systems concerning prognosis, with a higher C-index and a lower AIC. This result was confirmed in the subgroup of patients with $<16$ LNs examined. However, when LN status was modeled as a continuous variable, the LNR proved to be almost equivalent to the LODDS score, but when stratified by the TLNE, the LNP showed better predictive value.

Our results are consistent with those of previous authors. Lee et al. proved the LNR to be a better predictor for survival than the N category of the UICC/AJCC staging system because the LNR formula includes the TLNE[30]. Spolverato et al. found that the LODDS score was the best predictor of survival when assessed as a continuous variable; however, the LNR became the best predictor when LN status was included[31]. Liu et al. found the LODDS score and LNR to have almost equal predictive ability when assessed as continuous variables, but once categorized and clustered in the TLODDS staging system, the LODDS system lost part of 
its predictive value, whereas the LNR system did not[17]. Unlike these studies, Xu et al. analyzed 1302 patients with Siewert type II AEG who underwent preoperative radiotherapy and concluded that the LODDS score had higher predictive accuracy than the LNP and LNR[28]. These inconsistent results may be caused by the categorical cutoff values chosen. However, this study lacks information on indicators analyzed as continuous variables, which is necessary for directly comparing results from different studies[32].

A key aspect of the evaluation of a new marker is whether it provides additional information. The LNR uses the number of retrieved LNs as the denominator, and the LODDS score takes into account the number of negative LNs. Both of the newly added elements offer information on the extent of LN dissection, on the accuracy of the pathologic stage and, possibly, on the immune status of the patients[33, 34]. This can theoretically explain why the LNR and LODDS score are superior to the TNM pN system. We further illustrated the relationship between the LNR and LODDS scoring system through scatter plots. We found that the overall trend of the LNR and LODDS score is consistent and that the LODDS score is heterogeneous when the LNR is approximately 0 or 1 . Therefore, theoretically, the LODDS score has better discriminatory power for patients with a very low or high LNR[35-37]. Notably, there were only 9 patients (6\%) with $100 \%$ metastatic LNs in the current study, and therefore, the theoretical advantage of the LODDS score may be weakened. Categorization enables researchers to avoid strong assumptions about the relation between the marker and risk but at the expense of losing information[32]. We further compared the three staging systems when they were treated as categorical variables. Overall, each LNR stage is more evenly distributed than the other two staging systems. Moreover, the LODDS stage is more dependent on the TLNE. For example, there is no stage 1 for the LODDS staging system unless TLNE $\geq 14$. This may explain why the LNR performs better than the LODDS staging system when treated as a categorical variable.

Despite the limitations mentioned above, the LODDS score still has theoretical potential to be a good factor for clinical use. The lack of standard cutoffs for the stages may be a major obstacle because the calculation used to determine the cutoff value is affected by multiple factors, such as countries, research institutions, patient numbers, and the average TLNE[38]. We also need to pay attention to the mathematical characteristics of the LODDS score itself, which is calculated by adding a value of 0.5 to both the numerator and denominator. There is no proof that 0.5 is optimal for such an assessment[9]. As our data were obtained from a single institution, the current study may not reflect the status in other centers in China. Further research requires a better understanding of the LODDS score from a mathematical perspective and a better cutoff value, and thus, the LODDS score can be better used in clinical practice.

\section{Conclusion}

In conclusion, our data suggest that the phenomenon of stage migration is common among Siewert type II AEG patients. Our study also demonstrated that the TNM pN stage, LNR and LODDS systems appear to be independent prognostic factors in Siewert 2 AEG patients following curative resection. Specifically, the LNR represented the best prognostic factor when assessed as a categorical variable and that the LNR may serve as an alternative nodal staging system for AEG.

\section{Abbreviations}


AEG

Adenocarcinoma of the esophagogastric junction

LN

Lymph node

AJCC

American Joint Committee on Cancer

LNP

Positive lymph node

TNLE

Total number of lymph nodes examined

TNM

Tumor-node-metastasis

LNR

Lymph node ratio

LODDS

Log odds of metastatic lymph nodes

OS

Overall survival

C-index

Harrell's C-index statistic

AIC

Akaike information criterion

SD

Standard deviation

\section{Declarations}

\section{Ethics approval and consent to participate}

This research study was conducted retrospectively from data obtained for clinical purposes. We consulted extensively with the IRB of Southern Medical University who determined that our study did not need ethical approval. Informed consent was obtained from all individual participants included in the study.

\section{Consent for publication}

Not applicable.

\section{Availability of data and material}

The datasets used and/or analysed during the current study are available from the corresponding author on reasonable request. 


\section{Competing interests}

The authors declare that they have no competing interests

\section{Funding}

Not applicable.

\section{Authors' contributions}

Weilin Zhang is the first author because he contributed to this manuscript regarding data collection, statistical analysis, manuscript writing and formatting. Yong $\mathrm{Li}$ is the corresponding because he contributed to the conception of the study, helped perform the analysis with constructive discussions. All authors read and approved the final manuscript.

\section{References}

1. Brown LM, Devesa SS, Chow W-H. Incidence of Adenocarcinoma of the Esophagus Among White Americans by Sex, Stage, and Age. JNCI J Natl Cancer Inst. 2008;100:1184-7.

2. Dikken JL, Lemmens VE, Wouters MWJM, Wijnhoven BP, Siersema PD, Nieuwenhuijzen GA, et al. Increased incidence and survival for oesophageal cancer but not for gastric cardia cancer in the Netherlands. Eur J Cancer. 2012.

3. Liu K, Yang K, Zhang W, Chen X, Chen X, Zhang B, et al. Changes of esophagogastric junctional adenocarcinoma and gastroesophageal reflux disease among surgical patients during 1988-2012: A single-institution, high-volume experience in China. Ann Surg. 2016;263:88-95.

4. Maric R, Cheng KK. Classification of adenocarcinoma of the oesophagogastric junction. Br J Surg. 1999;86:1098-9.

5. Martin JT, Mahan A, Zwischenberger JB, McGrath PC, Tzeng CWD. Should gastric cardia cancers be treated with esophagectomy or total gastrectomy? A comprehensive analysis of 4,996 NSQIP/SEER patients. J Am Coll Surg. 2015;220:510-20.

6. Amin MB, Greene FL, Edge SB, Compton CC, Gershenwald JE, Brookland RK, et al. The Eighth Edition AJCC Cancer Staging Manual: Continuing to build a bridge from a population-based to a more "personalized" approach to cancer staging. CA Cancer J Clin. 2017;67:93-9.

7. Tan Z, Ma G, Yang H, Zhang L, Rong T, Lin P. Can lymph node ratio replace pn categories in the tumornode-metastasis classification system for esophageal cancer? J Thorac Oncol. 2014.

8. Kong SH, Lee HJ, Ahn HS, Kim JW, Kim WH, Lee KU, et al. Stage migration effect on survival in gastric cancer surgery with extended lymphadenectomy: The reappraisal of positive lymph node ratio as a proper N-staging. Ann Surg. 2012. 
9. Szczepanik AM, Paszko A, Szura M, Scully-Horner T, Kulig J. Alternative staging of regional lymph nodes in gastric cancer. Prz Gastroenterol. 2016;11:145-9.

10. Inoue K, Nakane Y, liyama H, Sato M, Kanbara T, Nakai K, et al. The superiority of ratio-based lymph node staging in gastric carcinoma. Ann Surg Oncol. 2002.

11. Sun Z, Xu Y, Li DM, Wang ZN, Zhu GL, Huang BJ, et al. Log odds of positive lymph nodes: A novel prognostic indicator superior to the number-based and the ratio-based $n$ category for gastric cancer patients with R0 resection. Cancer. 2010;116:2571-80.

12. Okusa T, Nakane Y, Boku T, Takada H, Yamamura M, Hioki K, et al. Quantitative analysis of nodal involvement with respect to survival rate after curative gastrectomy for carcinoma. Surg Gynecol Obstet. 1990;170:488-94.

13. Sun Z, Xu Y, Li DM, Wang ZN, Zhu GL, Huang BJ, et al. Log odds of positive lymph nodes: A novel prognostic indicator superior to the number-based and the ratio-based $n$ category for gastric cancer patients with R0 resection. Cancer. 2010;116:2571-80.

14. Cao J, Yuan P, Ma H, Ye P, Wang Y, Yuan X, et al. Log Odds of Positive Lymph Nodes Predicts Survival in Patients After Resection for Esophageal Cancer. Ann Thorac Surg. 2016;102:424-32.

15. Chen L-J, Chung K-P, Chang Y-J, Chang Y-J. Ratio and log odds of positive lymph nodes in breast cancer patients with mastectomy. Surg Oncol. 2015;24:239-47.

16. Petrucciani N, Carra MC, Martínez-Pérez A, Vitali GC, Landi F, Genova P, et al. Comparison of Different Nodal Staging in Patients with Locally Advanced Mid-low Rectal Cancer after Long-term Neoadjuvant Chemoradiation Therapy. Anticancer Res. 2019;39:2113-20.

17. Liu H, Deng J, Zhang R, Hao X, Jiao X, Liang H. The RML of lymph node metastasis was superior to the LODDS forevaluating the prognosis of gastric cancer. Int J Surg. 2013.

18. Smith DD, Nelson RA, Schwarz RE. A comparison of five competing lymph node staging schemes in a cohort of resectable gastric cancer patients. Ann Surg Oncol. 2014;21:875-82.

19. Amin MB, Edge SB, Greene FL, Byrd DR, Brookland RK, Washington MK, et al., editors. AJCC Cancer Staging Manual. 8th edition. Cham: Springer International Publishing; 2017.

20. Association JGC, others. Japanese Classification of Gastric Carcinoma - 2nd English Edition -. Gastric Cancer. 1998;1:0010.

21. Zhang H, Shang X, Chen C, Gao Y, Xiao X, Tang P, et al. Lymph node ratio-based staging system as an alternative to the current TNM staging system to assess outcome in adenocarcinoma of the esophagogastric junction after surgical resection. Oncotarget. 2016;7:74337-49.

22. Jian-hui C, Shi-rong C, Hui W, Si-le C, Jian-bo X, Er-tao Z, et al. Prognostic value of three different lymph node staging systems in the survival of patients with gastric cancer following D2 lymphadenectomy. Tumor Biol. 2016;37:11105-13.

23. Rao SJ. Regression Modeling Strategies: With Applications to Linear Models, Logistic Regression, and Survival Analysis. J Am Stat Assoc. 2003;98:257-8.

24. Chaurasia A, Harel O. Using AIC in multiple linear regression framework with multiply imputed data. Heal Serv Outcomes Res Methodol. 2012;12:219-33. 
25. Mariette C, Piessen G, Briez N, Gronnier C, Triboulet JP. Oesophagogastric junction adenocarcinoma: which therapeutic approach? Lancet Oncol. 2011;12:296-305.

26. Goto H, Tokunaga M, Miki Y, Makuuchi R, Sugisawa N, Tanizawa Y, et al. The optimal extent of lymph node dissection for adenocarcinoma of the esophagogastric junction differs between Siewert type II and Siewert type III patients. Gastric Cancer. 2015;18:375-81.

27. Curtis NJ, Noble F, Bailey IS, Kelly JJ, Byrne JP, Underwood TJ. The relevance of the Siewert classification in the era of multimodal therapy for adenocarcinoma of the gastro-oesophageal junction. J Surg Oncol. 2014;109:202-7.

28. Xu J, Cao J, Wang L, Wang Z, Wang Y, Wu Y, et al. Prognostic performance of three lymph node staging schemes for patients with Siewert type II adenocarcinoma of esophagogastric junction. Sci Rep. 2017;7:10123.

29. Liu K, Yang K, Zhang W, Chen X, Chen X, Zhang B, et al. Changes of Esophagogastric Junctional Adenocarcinoma and Gastroesophageal Reflux Disease Among Surgical Patients During 1988-2012. Ann Surg. 2016;263:88-95.

30. Lee JH, Kang J-W, Nam B-H, Cho GS, Hyung WJ, Kim MC, et al. Correlation between lymph node count and survival and a reappraisal of lymph node ratio as a predictor of survival in gastric cancer: A multiinstitutional cohort study. Eur J Surg Oncol. 2017;43:432-9.

31. Spolverato G, Ejaz A, Kim Y, Squires MH, Poultsides G, Fields RC, et al. Prognostic Performance of Different Lymph Node Staging Systems After Curative Intent Resection for Gastric Adenocarcinoma. Ann Surg. 2015;262:991-8.

32. Altman DG, Lausen B, Sauerbrei W, Schumacher M. Dangers of using "optimal" cutpoints in the evaluation of prognostic factors. J Natl Cancer Inst. 1994.

33. Siewert JR, Böttcher K, Stein HJ, Roder JD. Relevant Prognostic Factors in Gastric Cancer. Ann Surg. 1998;228:449-61.

34. Bando E. Outcome of Ratio of Lymph Node Metastasis in Gastric Carcinoma. Ann Surg Oncol. 2002;9:775-84.

35. Pei J-P, Zhang C-D, Fan Y-C, Dai D-Q. Comparison of Different Lymph Node Staging Systems in Patients With Resectable Colorectal Cancer. Front Oncol. 2019;8 JAN:1-8.

36. Aurello P, Petrucciani N, Nigri GR, La Torre M, Magistri P, Tierno S, et al. Log Odds of Positive Lymph Nodes (LODDS): What Are Their Role in the Prognostic Assessment of Gastric Adenocarcinoma? J Gastrointest Surg. 2014;18:1254-60.

37. La Torre M, Nigri G, Petrucciani N, Cavallini M, Aurello P, Cosenza G, et al. Prognostic assessment of different lymph node staging methods for pancreatic cancer with RO resection: pN staging, lymph node ratio, log odds of positive lymph nodes. Pancreatology. 2014;14:289-94.

38. Jin S, Wang J, Shen Y, Gan H, Xu P, Wei Y, et al. Comparison of different lymph node staging schemes in prostate cancer patients with lymph node metastasis. Int Urol Nephrol. 2020;52:87-95.

\section{Tables}


Table 1 Univariate analysis of survival determinants in patients with Siewert type II adenocarcinoma of the esophagogastric junction 


\begin{tabular}{|c|c|c|c|}
\hline Variable & No. of patients (\%) & Median survival time & P value \\
\hline \multicolumn{4}{|l|}{ Sex } \\
\hline Male & $111(70.7)$ & 42 & 0.574 \\
\hline Female & $46(29.3)$ & 37 & \\
\hline \multicolumn{3}{|l|}{ Age (years) } & \\
\hline$>65$ & $58(36.9)$ & 31 & 0.007 \\
\hline$\leq 65$ & $99(63.1)$ & 48 & \\
\hline \multicolumn{4}{|l|}{ Tumor size $(\mathrm{cm})$} \\
\hline$>3$ & $123(40.1)$ & 37 & 0.021 \\
\hline$\leq 3$ & $34(59.9)$ & 73 & \\
\hline \multicolumn{4}{|l|}{ Grade of differentiation } \\
\hline $\begin{array}{l}\text { Well or moderately } \\
\text { differentiated }\end{array}$ & $94(59.9)$ & 49 & 0.018 \\
\hline Poorly or undifferentiated undifferentiated & $63(40.1)$ & 31 & \\
\hline \multicolumn{4}{|l|}{ TNLE } \\
\hline$\geq 16$ & $86(54.8)$ & 44 & 0.015 \\
\hline$<16$ & $71(45.2)$ & 36 & \\
\hline \multicolumn{4}{|l|}{ Gastrectomy resection } \\
\hline Subtotal & $98(37.6)$ & 42 & 0.611 \\
\hline Total & $59(62.4)$ & 37 & \\
\hline Lymphovascular invasion & & & \\
\hline
\end{tabular}




\begin{tabular}{|c|c|c|c|}
\hline Present & $61(38.9)$ & 19 & 0.000 \\
\hline Absent & $96(61.1)$ & 49 & \\
\hline \multicolumn{4}{|c|}{ pT stage ${ }^{b}$} \\
\hline $\mathrm{T} 1$ & $1(0.6)$ & & 0.000 \\
\hline T2 & $24(15.3)$ & & \\
\hline T3 & $115(73.2)$ & 37 & \\
\hline T4 & $17(10.8)$ & 16 & \\
\hline \multicolumn{4}{|c|}{ Chemotherapy } \\
\hline Yes & $67(57.3)$ & 32 & 0.684 \\
\hline No & $90(42.7)$ & 43 & \\
\hline \multicolumn{4}{|c|}{ pN stage ${ }^{b}$} \\
\hline No & $42(26.8)$ & 69 & 0.000 \\
\hline N1 & $46(29.3)$ & 36 & \\
\hline N2 & $34(21.7)$ & 32 & \\
\hline N3 & $35(22.3)$ & 18 & \\
\hline \multicolumn{4}{|l|}{ LNR } \\
\hline 0 & $42(26.8)$ & 69 & 0.000 \\
\hline$\leq 0.3$ & $18(11.5)$ & 41 & \\
\hline$\leq 0.6$ & $43(27.4)$ & 49 & \\
\hline$>0.6$ & $54(33.4)$ & 17 & \\
\hline \multicolumn{4}{|l|}{ LODDS } \\
\hline$\leq-1.5$ & $24(15.3)$ & 69 & 0.000 \\
\hline$\leq-1$ & $25(15.9)$ & 68 & \\
\hline$\leq 0$ & $81(51.6)$ & 36 & \\
\hline
\end{tabular}

a Univariate Kaplan-Meier analysis. b According to the $8^{\text {th }}$ edition of the UICC/AJCC cancer staging manual. LN, lymph node; LNR, lymph node ratio; LODDS, log odds of positive lymph nodes; TNLE, total number of LNs examined 
Table 2 Predictors of survival in different lymph node staging systems on multivariate analysis by the Cox proportional hazards regression model 


\begin{tabular}{|c|c|c|c|c|c|c|c|c|c|}
\hline \multirow[t]{2}{*}{ Variable } & \multicolumn{3}{|c|}{ TNM pN } & \multicolumn{3}{|l|}{ LNR } & \multicolumn{3}{|c|}{ LODDS } \\
\hline & $\mathrm{HR}$ & $95 \% \mathrm{Cl}$ & $\mathrm{P}$ & $\mathrm{HR}$ & $95 \% \mathrm{Cl}$ & $P$ & $\mathrm{HR}$ & $95 \% \mathrm{Cl}$ & $\mathrm{P}$ \\
\hline $\begin{array}{l}\text { Grade of } \\
\text { differentiation }\end{array}$ & 1.265 & $\begin{array}{l}0.765- \\
2.093\end{array}$ & 0.360 & 0.937 & $\begin{array}{l}0.559- \\
1.572\end{array}$ & 0.806 & 1.151 & $\begin{array}{l}0.694- \\
1.909\end{array}$ & 0.586 \\
\hline Tumor size & 1.819 & $\begin{array}{l}1.094- \\
3.023\end{array}$ & 0.021 & 1.758 & $\begin{array}{l}1.061- \\
2.912\end{array}$ & 0.029 & 1.905 & $\begin{array}{l}1.140- \\
3.184\end{array}$ & 0.014 \\
\hline Age & 2.343 & $\begin{array}{l}1.506- \\
3.644\end{array}$ & 0.000 & 2.038 & $\begin{array}{l}1.293- \\
3.212\end{array}$ & 0.002 & 2.314 & $\begin{array}{l}1.493- \\
3.587\end{array}$ & 0.000 \\
\hline $\begin{array}{l}\text { Lymphovascular } \\
\text { invasion }\end{array}$ & 0.608 & $\begin{array}{l}0.365- \\
1.012\end{array}$ & 0.056 & 0.660 & $\begin{array}{l}0.383- \\
1.037\end{array}$ & 0.069 & 0.600 & $\begin{array}{l}0.359- \\
1.002\end{array}$ & 0.051 \\
\hline pT degree & 1.908 & $\begin{array}{l}1.255- \\
2.903\end{array}$ & 0.003 & 2.050 & $\begin{array}{l}1.349- \\
3.115\end{array}$ & 0.001 & 2.151 & $\begin{array}{l}1.417- \\
3.267\end{array}$ & 0.000 \\
\hline TNLE & 0.488 & $\begin{array}{l}0.306- \\
0.779\end{array}$ & 0.003 & 0.444 & $\begin{array}{l}0.288- \\
0.683\end{array}$ & 0.000 & 0.483 & $\begin{array}{l}0.305- \\
0.763\end{array}$ & 0.002 \\
\hline pN stage & & & 0.001 & & & & & & \\
\hline \multicolumn{10}{|l|}{ NO (reference) } \\
\hline N1 & 2.459 & $\begin{array}{l}1.338- \\
4.521\end{array}$ & 0.004 & & & & & & \\
\hline N2 & 2.154 & $\begin{array}{l}1.087- \\
4.269\end{array}$ & 0.028 & & & & & & \\
\hline N3 & 3.477 & $\begin{array}{l}1.841- \\
6.565\end{array}$ & 0.000 & & & & & & \\
\hline LNR stage & & & & & & 0.000 & & & \\
\hline \multicolumn{10}{|l|}{$\begin{array}{l}\text { LNRO } \\
\text { (reference) }\end{array}$} \\
\hline LNR1 & & & & 2.437 & $\begin{array}{l}1.088- \\
5.458\end{array}$ & 0.030 & & & \\
\hline LNR2 & & & & 1.553 & $\begin{array}{l}0.824- \\
2.927\end{array}$ & 0.174 & & & \\
\hline LNR3 & & & & 4.174 & $\begin{array}{l}2.327- \\
7.485\end{array}$ & 0.000 & & & \\
\hline LODDS stage & & & & & & & & & 0.007 \\
\hline \multicolumn{10}{|l|}{$\begin{array}{l}\text { LODDS1 } \\
\text { (reference) }\end{array}$} \\
\hline LODDS2 & & & & & & & 0.614 & $\begin{array}{l}0.236- \\
1.596\end{array}$ & 0.317 \\
\hline LODDS3 & & & & & & & 1.463 & $\begin{array}{l}0.720- \\
2.973\end{array}$ & 0.293 \\
\hline
\end{tabular}


LN, lymph node; LNR, lymph node ratio; LODDS, log odds of positive lymph nodes; TNLE, total number of LNs examined.

\section{Table 3 Comparison of the prognostic value of the different lymph node staging systems with different TNLE}

\begin{tabular}{|lllll|}
\hline Variable & \multicolumn{1}{l}{ TNLE } & & & \\
\cline { 2 - 5 } & ALL & & $<16$ & \\
\cline { 2 - 5 } & C-index & AIC & C-index & AIC \\
\hline LNP & $0.721(0.670-0.773)$ & 946.935 & $0.809(0.755-0.865)$ & 350.265 \\
\hline LNR (continuous) & $0.725(0.674-0.776)$ & 938.918 & $0.793(0.738-0.848)$ & 353.971 \\
\hline LODDS (continuous) & $0.729(0.680-0.778)$ & 940.483 & $0.785(0.725-0.846)$ & 356.302 \\
\hline TNM pN & $0.740(0.693-0.788)$ & 934.349 & $0.802(0.742-0.863)$ & 354.773 \\
\hline LNR (category) & $0.752(0.706-0.799)$ & 926.350 & $0.810(0.752-0.870)$ & 352.293 \\
\hline LODDS (category) & $0.737(0.686-0.786)$ & 939.087 & $0.792(0.730-0.856)$ & 358.493 \\
\hline
\end{tabular}

C-index, Harrell's concordance index; AIC, Akaike information criterion; LN, lymph node; LNP, number of positive LNs; LODDS, log odds of positive lymph nodes; LNR, lymph node ratio; TNLE, total number of LNs examined.

\section{Table 4 Spearman's correlation test between the number of TNLE, positive LNs, LNR value and LODDS value}

\begin{tabular}{|lllllll|}
\hline Variable & Positive LN & LNR & LODDS & Variable & Positive LN & LNR \\
& r & P & r & P & $r$ & P \\
\hline TNLE & 0.198 & 0.013 & -0.075 & 0.349 & 0.198 & 0.013 \\
\hline LNP & NA & NA & 0.927 & 0.000 & 0.887 & 0.000 \\
\hline LNR & 0.927 & 0.000 & NA & NA & 0.965 & 0.000 \\
\hline
\end{tabular}


LN, lymph node; LNR, lymph node ratio; LODDS, log odds of positive lymph nodes; LNP, number of positive LNs; TNLE, total number of LNs examined; NA, not available.

\section{Figures}

A
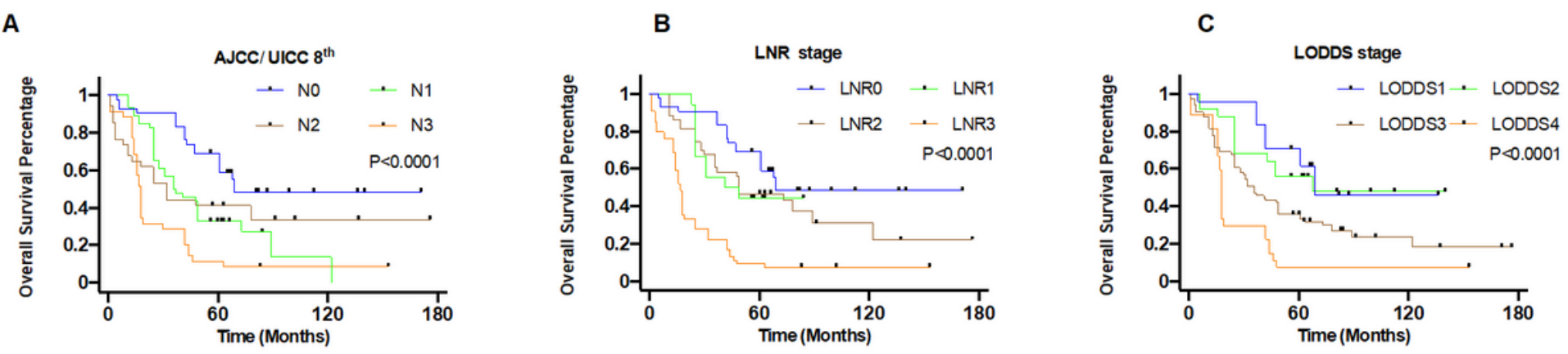

Figure 1

Kaplan-Meier curves for Overall Survival stratified by TNM pN categories based on the AJCC/UICC 8th (A), LNR (B), and LODDS (C) systems

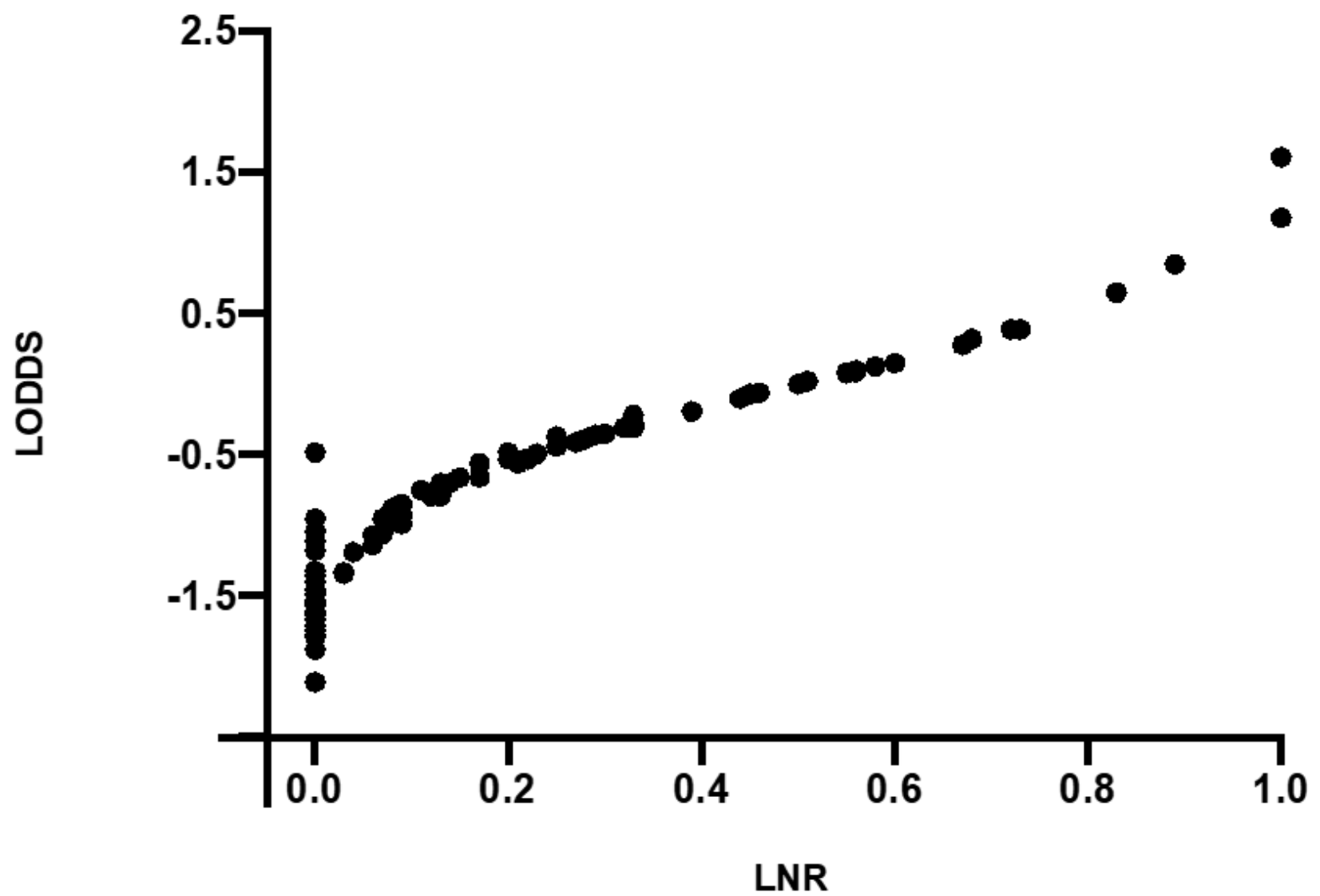

Figure 2

Scatter plot of the LNR and LODDS score 
TNM pN
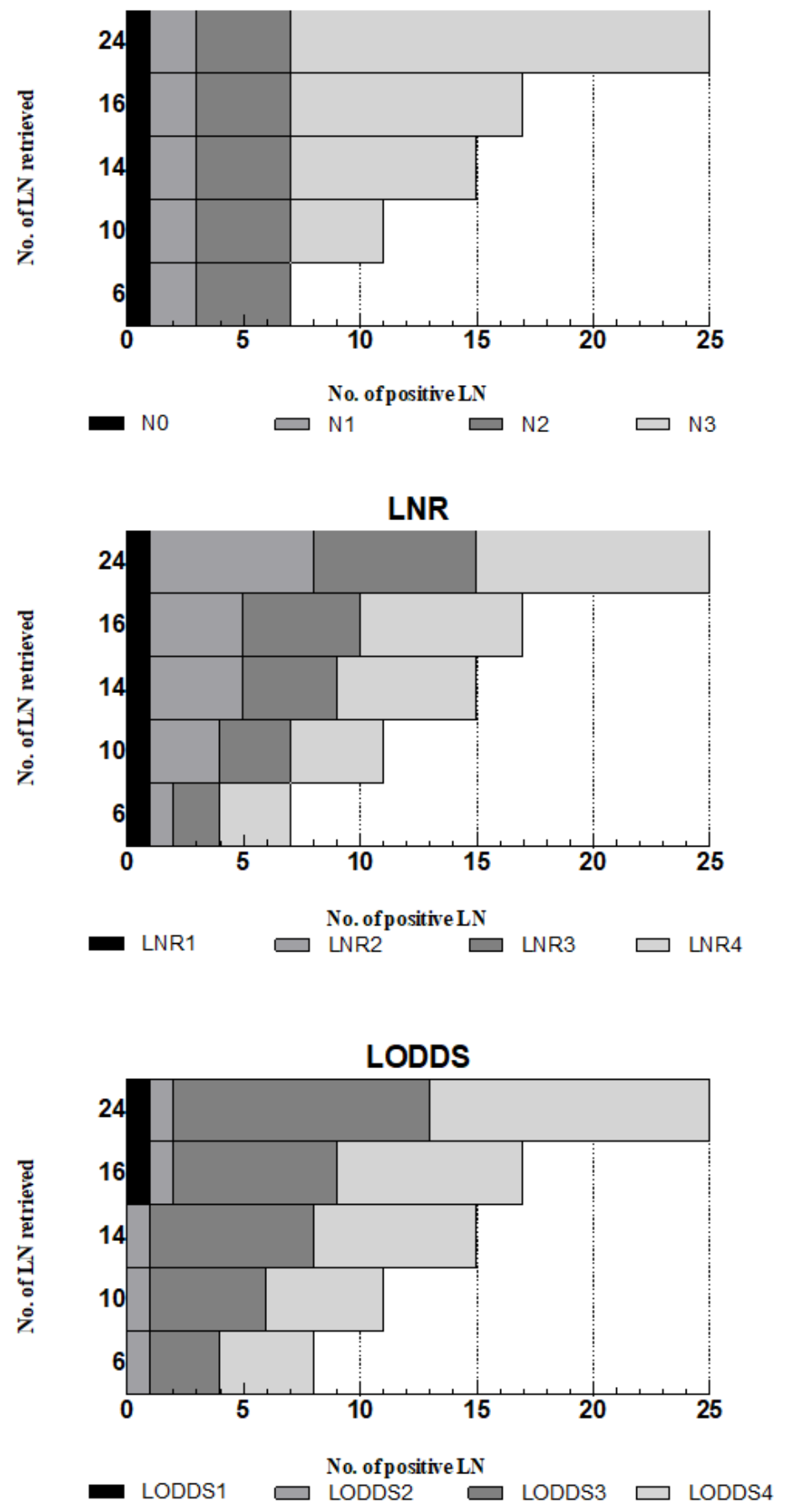

Figure 3

Comparison of the TNM pN, LNR and LODDS staging systems 